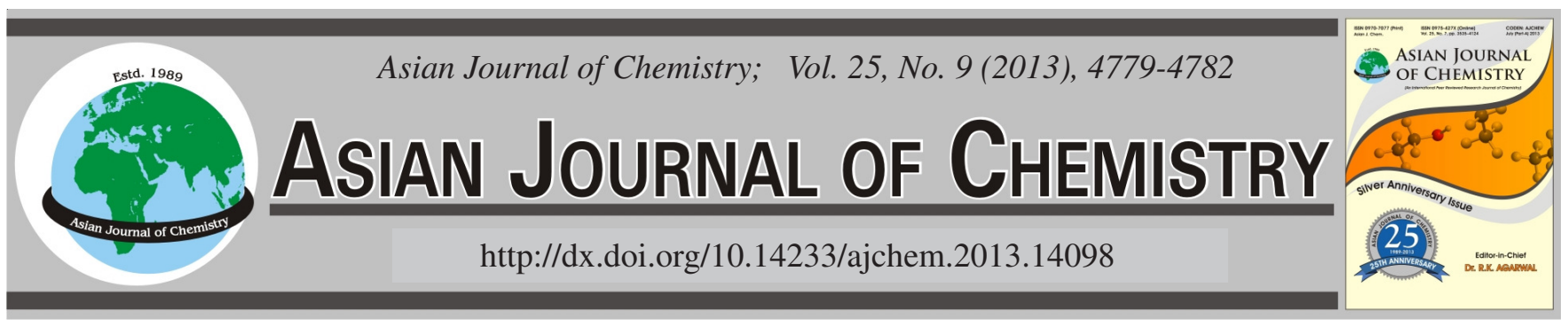

\title{
Study of Preparation and Thermal Stability of Cyano-Functionalized Imidazolium Type Ionic Liquids
}

\author{
W.D. LIANG ${ }^{1,2,3,}$, H.F. $\mathrm{Li}^{3}$, G.J. Gou ${ }^{3}$ and A.Q. WANG ${ }^{1}$
}

${ }^{1}$ Lanzhou Institute of Chemical Physics, Chinese Academy of Sciences, Lanzhou, P.R. China

${ }^{2}$ Graduate School of Chinese Academy of Sciences, Beijing, P.R. China

${ }^{3}$ School of Petrochemical Engineering, Lanzhou University of Technology, Lanzhou, P.R. China

*Corresponding author: Fax: +86931 2973741; Tel: +86931 2973383; E-mail: davidlucas@163.com

\begin{abstract}
Sixteen imidazolium type cyano-functionalized Brønsted acidic ionic liquids with $\mathrm{HSO}_{4}{ }^{-}$and $\mathrm{H}_{2} \mathrm{PO}_{4}{ }^{-}$anion were prepared adopting twostep method and were characterized by FT-IR, ${ }^{1} \mathrm{H}$ and ${ }^{13} \mathrm{C}$ NMR. The onset temperatures of thermal decomposition of the ionic liquids were measured using thermogravimetric analysis. It was found that the decomposition onset temperatures of the as-synthesized ionic liquids were in the range of $200-260^{\circ} \mathrm{C}$. Additionally, the decomposition temperatures were highly dependent on the nature of the anions. Thermal stability of these cyano-functionalized ionic liquids decreased in the order of $\mathrm{HSO}_{4}^{-}>\mathrm{H}_{2} \mathrm{PO}_{4}^{-}$.

Key Words: Brønsted acid, Ionic liquid, Cyano-functionalized, Thermal stability, Imidazolium.
\end{abstract}

\section{INTRODUCTION}

There are a growing awareness of research in the field of ionic liquids ${ }^{1-3}$ due to their particular properties, such as nonflammability, insignificant vapour pressure and wide electrochemical window, which make them suitable candidates for a wide variety of applications. Ionic liquids based on $\mathrm{AlCl}_{3}$ appeared the earliest that are unstable and difficult for reutilization. Therefore, non-chloroaluminiate ionic liquids, which are air and moisture stable, have been developed and successfully applied to synthesis, extraction, separation, etc. With the development of ionic liquids study, which contain exclusively alkyl functional group cannot meet specific demands. Thus a novel class of task specific ionic liquids (TSILs) containing special functional group with cations or anions have been developed ${ }^{4}$. The specific functional groups include hydroxyl group ${ }^{5}$, carboxyl group ${ }^{6}$, ester group ${ }^{7}$, cyano group $^{8}$, amino group ${ }^{9}$, chiral group ${ }^{10}$.

As the application of ionic liquids in high-temperature systems has progressively increased, thermal stability of ionic liquids has become a significant property. The thermal properties of alkyl imidazolium-type ionic liquids are stable up to about $300{ }^{\circ} \mathrm{C}^{11}$. Amarasekara et al. ${ }^{12}$ studied the thermal stabilities of sulfonic acid group-functionalized ionic liquids and reported that the decomposition temperatures of these ionic liquids are highly dependent on the nature of the anion. Wooster et al. ${ }^{13}$ reported the long term thermal stability of ionic liquids containing cyano group. It has been reported that alkali melt as well as alkali melt oxides, quaternary ammonium alkali, which added more difficulty on result after-treatment ${ }^{14}$, play an important role in catalyzing cyanoethylation reaction ${ }^{15}$.

As far as we are aware, there is a lack of data concerning cyano-functionalized ionic liquids containing carboxyl, alcohol, long chain alkyl on the cation. In this paper, we report the synthesis of precursor without catalyst in order to obtain cyanofunctionalized Brønsted acidic ionic liquids (Fig. 1) and determine the thermal stability of these ionic liquids by thermogravimetric analysis (TGA).

\section{EXPERIMENTAL}

All chemicals are commercially available and used without further purification. FT-IR spectra were recorded on a Bruker EQUINOX FT-IR spectrometer using $\mathrm{KBr}$ pellets in the range of $5000-4000 \mathrm{~cm}^{-1},{ }^{1} \mathrm{H}$ and ${ }^{13} \mathrm{C}$ NMR spectra were obtained on a Mercury-400BB spectrometer at $400 \mathrm{MHz}$ in DMSO- $d_{6}$ solution. TGA was performed on a thermal analysis system (Seiko Instruments, STA 499C), which were run in alumina pan with a heating rate of $10^{\circ} \mathrm{C} / \mathrm{min}$ under argon atmosphere and the temperature was programmed from 25 $900{ }^{\circ} \mathrm{C}$.

Synthesis of precursor 1-(2-cyanoethyl)imidazole (1): In a three-necked, $250 \mathrm{~mL}$ round-bottomed flask equipped with reflux condenser and magnetic stirrer, $6.808 \mathrm{~g}(0.1 \mathrm{~mol})$ of imidazole was mixed with $30 \mathrm{~mL}$ methanol, then $7.25 \mathrm{~mL}$ 

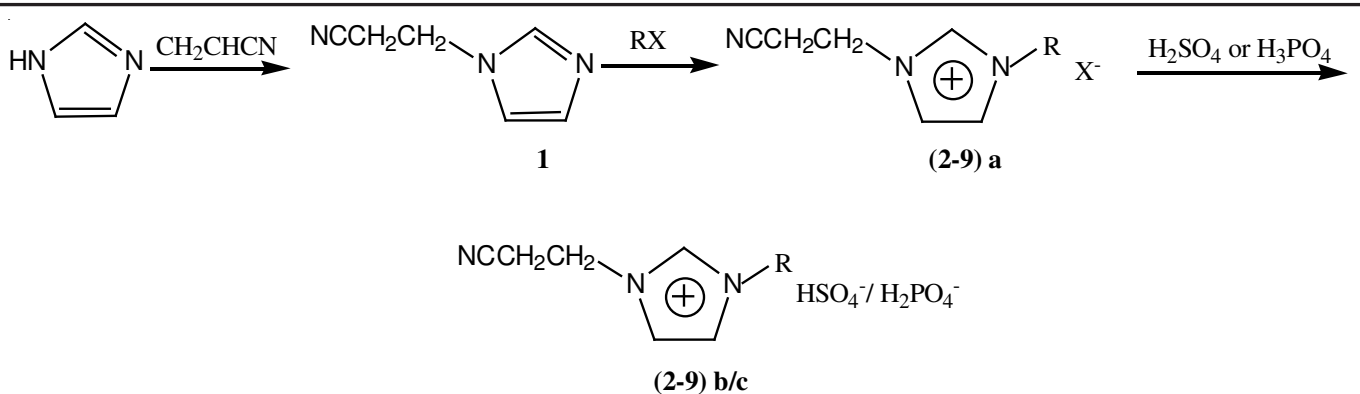

\author{
$\mathrm{R}=$ (2), $\mathrm{CH}_{2} \mathrm{COOH}(3), \mathrm{CH}_{2} \mathrm{CH}_{2} \mathrm{OH}(4), \mathrm{CH}_{2} \mathrm{CH}_{3}(5),\left(\mathrm{CH}_{2}\right)_{4} \mathrm{CH}_{3}(6),\left(\mathrm{CH}_{2}\right)_{5} \mathrm{CH}_{3}(7),\left(\mathrm{CH}_{2}\right)_{6} \mathrm{CH}_{3}(\mathbf{8}),\left(\mathrm{CH}_{2}\right)_{7} \mathrm{CH}_{3}(9)$ \\ $\mathrm{X}=\mathrm{Cl}, \mathrm{Br}$
}

Fig. 1. Synthetic pathway to functional Brønsted acidic ionic liquids

(0.11 mol) of acrylonitrile was dropped into the solution over a period of $0.5 \mathrm{~h}$ at $20-30^{\circ} \mathrm{C}$. The solution was heated rapidly to $60{ }^{\circ} \mathrm{C}$ under stirring for an additional period of $6 \mathrm{~h}$. Finally, the solvent and residual acrylonitrile was removed under reduced pressure. A pale yellow liquid was obtained with a yield of $97.3 \%(11.783 \mathrm{~g})$.

Synthesis of intermediates (2-9)a: The synthesis of 1-(2-cyanoethyl)-3- benzylimidazolium chloride ([cebzim]Cl) (2a) was carried out in a $250 \mathrm{~mL}$ round-bottomed flask, which was immersed in a recirculating heated oil-bath and fitted with a reflux condenser. $3.635 \mathrm{~g}$ (30 mmol) 1-(2-cyanoethyl)imidazole was dissolved in $20 \mathrm{~mL}$ of acetonitrile. Then 3.5 $\mathrm{mL}(33 \mathrm{mmol})$ of freshly distilled benzyl chloride was added with constant stirring at $40{ }^{\circ} \mathrm{C}$ and refluxed for $6 \mathrm{~h}$. Finally, the reaction mixture was distilled to remove the solvent. The molten salt was repeatedly washed with absolute ether and distilled at $50{ }^{\circ} \mathrm{C}$ to yield objective product as a yellowish oil liquid. And the pure product was obtained by column chromatography (petroleum ether:ethyl acetate $=6: 1$ ) as a light yellow liquid. The product was dried under vacuum for $24 \mathrm{~h}(6.986$ g, $94 \%)$.

Chloroacetic acid instead of benzyl chloride was used for preparation of 1-(2-cyanoethyl)-3-carboxylmethyl imidazolium chloride ([cecmim]Cl) (3a), 1-(2-cyanoethyl)-3-hydroxyethylimidazolium chloride ([ceheim]Cl) (4a) as a viscous liquid at $50^{\circ} \mathrm{C}$ under $\mathrm{N}_{2}$ atmosphere.

1-(2-Cyanoethyl)-3-alkylimidazolium bromides (5a-9a) were prepared as Gong and coworkers described ${ }^{16}$.

Synthesis of objective ionic liquids (2-9b/c): 1-(2Cyanoethyl)-3-benzylimidazolium hydrogen sulphate ([cebzim] $\mathrm{HSO}_{4}$ ) (2b) was synthesized as following: 1-(2Cyanoethyl)-3-benzylimidazoliolium chloride (30 mmol) was dissolved in $30 \mathrm{~mL}$ of absolute alcohol. Equal molar of sulfuric acid $(98 \%)$ was added dropwise with stirring in ice bath, which was warmed to $80{ }^{\circ} \mathrm{C}$ with sufficient reflux for $2 \mathrm{~h}$. The produced ionic liquid was washed repeatedly with diethyl ether and dried in a vacuum to afford [cebzim] $\mathrm{HSO}_{4}(\mathbf{2 b})$ as highly viscous and pale-yellow oily liquid (9.093 g, $98 \%$ ).

[cebzim] $\mathrm{H}_{2} \mathrm{PO}_{4}(\mathbf{2 c})$ was a viscous liquid and also prepared with a similar procedure by using phosphoric acid (85\%) instead of sulfuric acid (98\%). The other 14 kinds of ionic liquids (3-9b/c) were synthesized as [cebzim] $\mathrm{HSO}_{4}$ and [cebzim] $\mathrm{H}_{2} \mathrm{PO}_{4}$ and obtained as yellowish oily liquid (Table-1).

\begin{tabular}{cccccc}
\multicolumn{5}{c}{ TABLE-1 } \\
\multicolumn{7}{c}{$\begin{array}{c}\text { SPECIFIC TEMPERATURES, REACTION TIMES } \\
\text { AND YIELDS FOR COMPOUNDS (2-9)b/c }\end{array}$} \\
\hline Comp. & $\mathrm{R}$ & Anion & $\begin{array}{c}\text { Temp. } \\
\left({ }^{\circ} \mathrm{C}\right)\end{array}$ & $\begin{array}{c}\text { Reaction } \\
\text { time }(\mathrm{h})\end{array}$ & $\begin{array}{c}\text { Yield } \\
(\%)\end{array}$ \\
\hline $\mathbf{2 b}$ & bzl & & & 2 & 98 \\
$\mathbf{3 b}$ & Carboxylmethyl & & & 4 & 93 \\
$\mathbf{4 b}$ & Hydroxyethyl & & & 4 & 87 \\
$\mathbf{5 b}$ & Et & $\mathrm{HSO}_{4}^{-}$ & \multirow{2}{*}{80} & 6 & 93 \\
$\mathbf{6 b}$ & Pen & & & 6 & 97 \\
$\mathbf{7 b}$ & Hex & & & 8 & 99 \\
$\mathbf{8 b}$ & Hep & & & 10 & 95 \\
$\mathbf{9 b}$ & Oct & & & 10 & 99 \\
\hline $\mathbf{2 c}$ & Benzyl & & & 2 & 97 \\
$\mathbf{3 c}$ & Carboxylmethyl & & & 4 & 94 \\
$\mathbf{4 c}$ & Hydroxyethyl & & & 4 & 90 \\
$\mathbf{5 c}$ & Ethyl & $\mathrm{H}_{2} \mathrm{PO}_{4}^{-}$ & 80 & 6 & 89 \\
$\mathbf{6 c}$ & Pentyl & & & 8 & 96 \\
$\mathbf{7 c}$ & Hexyl & & & 8 & 92 \\
$\mathbf{8 c}$ & Heptyl & & & 10 & 99 \\
$\mathbf{9 c}$ & Octyl & & 10 & 98 \\
\hline
\end{tabular}

\section{RESULTS AND DISCUSSION}

The decomposition onset temperature data of the prepared cyano-functionalized Brønsted acidic ionic liquids are summarized in Table-2. They are thermally stable up to 200$260{ }^{\circ} \mathrm{C}$. It is evident that all of the $\mathrm{HSO}_{4}^{-}$based ionic liquids present higher thermal stability than that of $\mathrm{H}_{2} \mathrm{PO}_{4}^{-}$based ionic liquids, except for $\mathbf{9 b}$ which shows a distinct weight loss at $45^{\circ} \mathrm{C}$ and exhibits three peaks in the DTG curves different from the other ionic liquids that show one to two peaks. Therefore, it is suggested that their decompositions are highly dependent upon the anion associated with the ionic liquids. Meanwhile, all of the cyano-functionalized imidazolium hydrogen sulphates with the unsaturated side chains (bzl, carboxylmethyl) are thermally more stable than the corresponding ionic liquids with saturated side chains.

\begin{tabular}{ccccccccc}
\multicolumn{10}{c}{ TABLE-2 } \\
\multicolumn{10}{c}{$\begin{array}{c}\text { DECOMPOSITION ONSET TEMPERATURE } \\
\left({ }^{\circ} \mathrm{C}\right)\end{array}$} \\
\hline & $\mathbf{2}$ & $\mathbf{3}$ & $\mathbf{4}$ & $\mathbf{5}$ & $\mathbf{6}$ & $\mathbf{7}$ & $\mathbf{8}$ & $\mathbf{9}$ \\
\hline b & 260 & 220 & 218 & 219 & 212 & 218 & 217 & 45 \\
c & 200 & 202 & 203 & 210 & 205 & 202 & 204 & 203 \\
\hline
\end{tabular}

The order of thermal stability of ionic liquids $\mathbf{2 b}-\mathbf{4} \mathbf{c}$ was found to be $\mathbf{2 b}>\mathbf{3 b}>\mathbf{4 b}>\mathbf{2 c} \approx \mathbf{3} \mathbf{3} \approx \mathbf{4}$, as illustrated in Fig. 2 . 


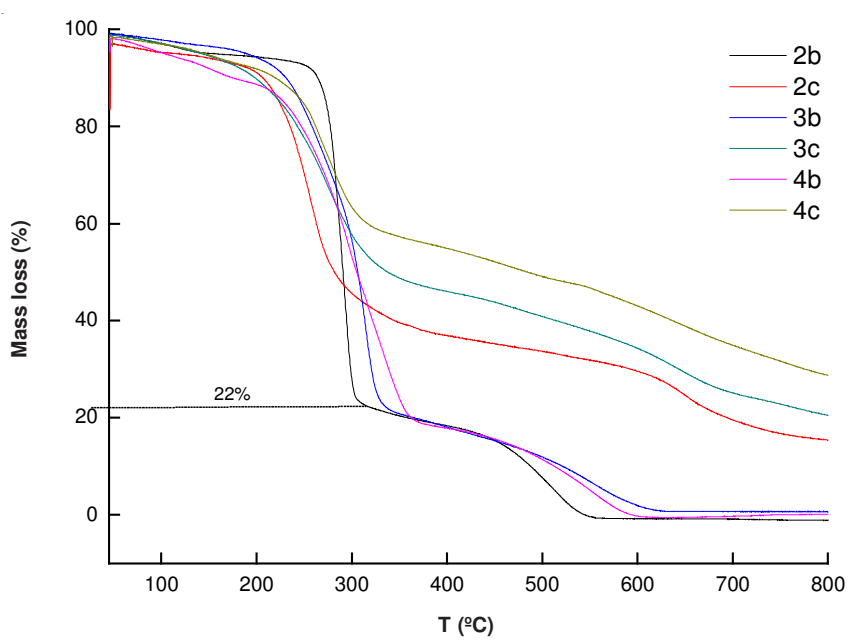

Fig. 2. Thermal gravimetric analysis of $\mathbf{2 b - 4 c}$

Obviously, the thermal stability of these compounds are highly dependent on the anion and decrease in the order $\mathrm{HSO}_{4}^{-}>$ $\mathrm{H}_{2} \mathrm{PO}_{4}^{-}$. Meanwhile, all of these ionic liquids present similar two-step decomposition behaviours. Herein, we make the explanation for the decomposable process of $\mathbf{2} \mathbf{b}$ as an example. [Cebzim $] \mathrm{H}_{2} \mathrm{PO}_{4}$ begins to decompose at $257^{\circ} \mathrm{C}$ and its mass loss is $78 \%$ when the temperature rises to $310^{\circ} \mathrm{C}$. Ultimately, decomposition is almost complete at $550{ }^{\circ} \mathrm{C}$ and come to a plateau. In addition, for the samples of $\mathbf{2 b}, \mathbf{3 b}$ and $\mathbf{4 b}$, which have a thorough decomposition at nearly $615^{\circ} \mathrm{C}$, whereas the samples of $\mathbf{2 c}, \mathbf{3 c}$ and $\mathbf{4 c}$ are not completely decomposed.

The TGA curves for alkylimidazolium type ionic liquids series with $\mathrm{HSO}_{4}{ }^{-}$and $\mathrm{H}_{2} \mathrm{PO}_{4}{ }^{-}$anion $(\mathrm{R}=$ alkyl) are shown in Figs. 3 and 4, respectively. However, the length of the alkyl chain has not a strong influence on decomposition onset temperature. It can be seen from Fig. 3 that decomposition onset temperatures for the samples of $\mathbf{5 b}, \mathbf{6 b}, \mathbf{7 b}$ and $\mathbf{8 b}$ are similar. The TGA measurement of $\mathbf{9 b}$ shows a distinct massloss of $c a .19 \%$ at $c a .200{ }^{\circ} \mathrm{C}$. The decomposition temperature decreases in the order of $\mathbf{8 b} \approx \mathbf{7 b} \approx \mathbf{6} b \approx \mathbf{5 b}>\mathbf{9 b}$. Interestingly, a somewhat close thermal stability is found in the alkylimidazolium type ionic liquids with $\mathrm{H}_{2} \mathrm{PO}_{4}{ }^{-}$anion, all of which are stable up to $c a .200{ }^{\circ} \mathrm{C}$, as represented in Fig. 4 .

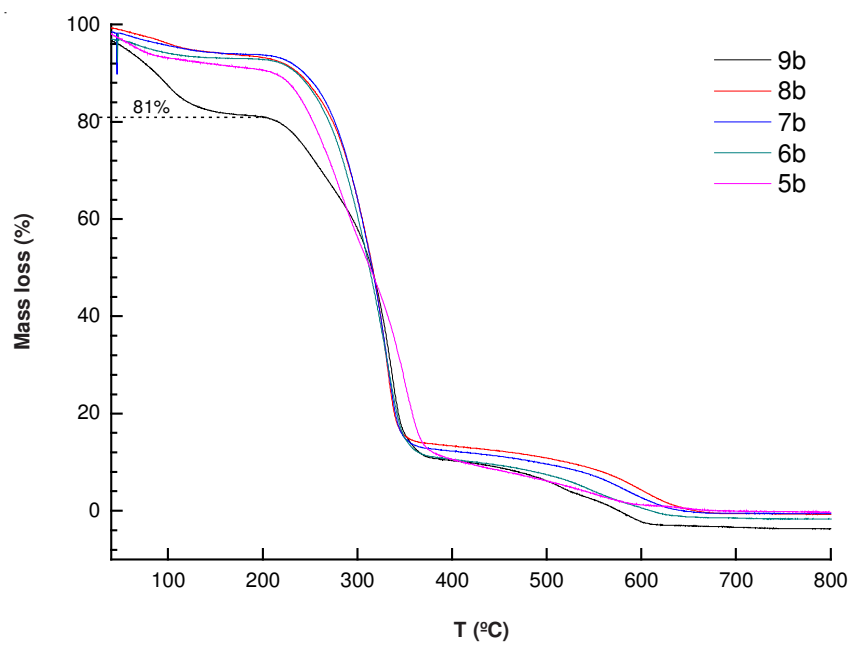

Fig. 3. Thermal gravimetric analysis (TGA) of (5-9)b

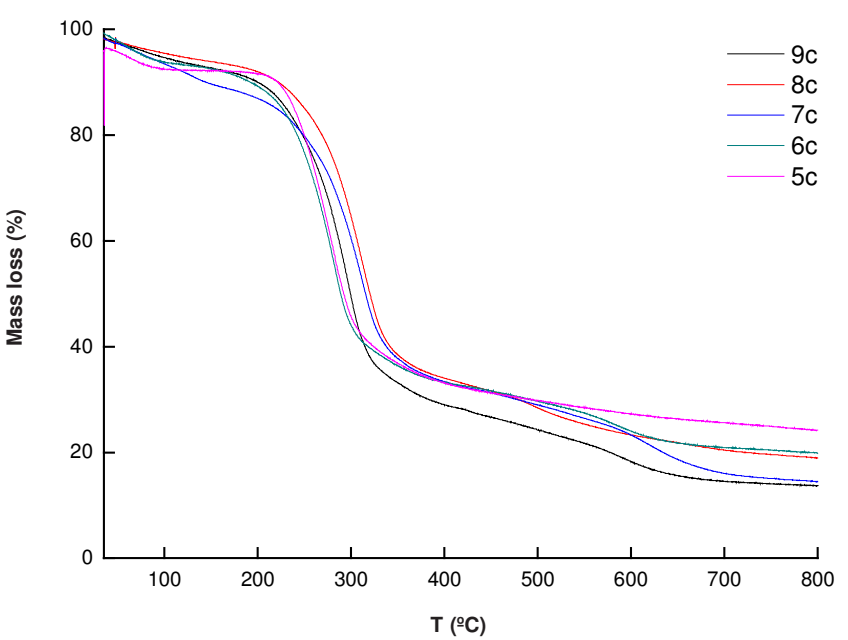

Fig. 4. Thermal gravimetric analysis of (5-9)c

Most of the cyano-functionalized ionic liquids are stable at or above $200^{\circ} \mathrm{C}$, which is low compared to alkylimidazolium based-ionic liquids (general $\mathrm{T}_{\mathrm{d}}>300^{\circ} \mathrm{C}^{11}$ ). The reason for it could be relative to lower symmetry of the functionalized imidazolium. In addition, cation size increases with the increasing of alkyl chain length, which leads to the weakening of gravity between cation and anion with the distance widening. Hence, ionic liquids decompose more easily in the process of heating, resulting in onset decomposition temperature drop ${ }^{17}$. Moreover, the thermal decomposition depends on the nature of anions. Further thermal decomposition also decreases with increasing hydrophilicity of anions ${ }^{18}$. The decomposition step was noticed to be steeper for an ionic liquid containing $\mathrm{HSO}_{4}{ }^{-}$ rather than $\mathrm{H}_{2} \mathrm{PO}_{4}^{-}$and all ionic liquids (2-9)b/c show twostage decomposition process in the TG curves, which may be due to the fact that their decomposition mechanisms are highly dependent on the nature of the anion.

\section{Conclusion}

Cyano-functionalized Brønsted acidic ionic liquids were successfully synthesized from the precursor of 1-(2-cyanoethyl)imidazole, which was prepared by cyanoethylation without catalyst. The decomposition onset temperatures of ionic liquids are in the range of $200-260{ }^{\circ} \mathrm{C}$ in this study. The main factors that influence the thermal stability of these ionic liquids are attributed to the structure of the corresponding anions and the thermal stability decreases in the order $\mathrm{HSO}_{4}^{-}>\mathrm{H}_{2} \mathrm{PO}_{4}^{-}$. The onset temperatures of ionic liquids are affected slightly by the size of the alkyl chain of the cation. Most of the salts studied exhibit apparently two-stage mass loss. In addition, all hydrogen sulphates containing the unsaturated side chains $(\mathrm{R}=\mathrm{bzl}$, carboxylmethyl) are thermally more stable compared to the corresponding ionic liquids with the saturated side chains. Herein, it is expected that the present work may provide the basic guidance for designing novel ionic liquids catalysts for acid catalytic reaction.

\section{REFERENCES}

1. K.N. Marsh and A. Deer, Chem. Eng., 19, 357 (2002).

2. L. Alonso, A. Arce, M. Francisco and A. Soto, J. Chem. Eng. Data, 55, 3262 (2010).

3. U. Domanska and M. Królikowska, J. Chem. Eng. Data, 56, 124 (2011). 
4. X.H. Li, D.B. Zhao, Z.F. Fei and L.F. Wang, Sci. China B, 49, 385 (2006).

5. D. Dorjnamjin, M. Ariunaa and Y.K. Shim, Int. J. Molecul. Sci., 9, 807 (2008).

6. W.G. Geng, X.H. Li, L.F. Wang and H.L. Duan, Acta. Phys-Chim. Sin., 22, 230 (2006)

7. L.Y. Zhu, L.G. Chen, X. Zhou and Y. Fu, Speciality Petrochem., 26, 38 (2009).

8. A.I. Siriwardana, A.A.J. Torriero, J.M. Reyna-Gonzalez, I.M. Burgar, N.F. Dunlop, A.M. Bond, G.B. Deacon and D.R. MacFarlane, J. Org. Chem., 75, 8376 (2010).

9. B. Clare, A.R. Sirwardana and D.R. MacFarlane, Top. Curr. Chem., 90, 1 (2009).

10. J.C. Pastre, Y. Génisson, N. Saffon, J. Dandurand and C.R.D. Correia, J. Braz. Chem. Soc., 21, 821 (2010).
11. G.H. Min, T. Yim, H.Y. Lee, D.H. Huh, E. Lee, J. Mun, S.M. Oh and Y.G. Kim, Bull. Korean Chem. Soc., 27, 847 (2006).

12. A.S. Amarasekara and A.S. Owereh, J. Therm. Anal. Calorim., 103, 1027 (2011).

13. T.J. Wooster, K.M. Johanson, K.J. Fraser, D.R. MacFarlane and J.T. Scott, Green Chem., 8, 691 (2006).

14. Z.Y. Chen and H. Zhong, Chem. Eng., 92, 12 (2002).

15. C. Ramalingan, S. Balasubramanian and S. Kabflan, Eur. J. Med. Chem., 39, 527 (2004).

16. S.M. Gong, H.Y. Ma, X.H. Wan and Y.F. Zhao, Chem. J. Chin. Univ., 27, 761 (2006).

17. H.L. Ngo, K. LeCompte, L. Hargens and A.B. McEwen, Thermochim. Acta, 357, 97 (2000).

18. G. Singh and A. Kumar, Indian J. Chem., 47A, 495 (2008). 